\title{
Hierarchically structured electrospun scaffolds with chemically conjugated growth factor for ligament tissue engineering
}

Hannah M. Pauly, BE ${ }^{1}$, Binulal N. Sathy, $\mathrm{PhD}^{2}$, Dinorath Olvera, $\mathrm{BS}^{2}$, Helen O. McCarthy, $\mathrm{PhD}^{3}$, Daniel

J. Kelly, $\mathrm{PhD}^{2,4,5,6}$, Ketul C. Popat, $\mathrm{PhD}^{1,7}$, Nicholas J. Dunne, $\mathrm{PhD}^{2,3,8}$, Tammy L. Haut Donahue, $\mathrm{PhD}^{1,7}$ ${ }^{1}$ School of Biomedical Engineering, Colorado State University, Fort Collins, CO USA

${ }^{2}$ Trinity Centre for Bioengineering, Trinity Biomedical Sciences Institute, Trinity College Dublin, Dublin, Ireland. ${ }^{3}$ School of Pharmacy, Queen's University Belfast, Belfast, UK.

${ }^{4}$ Department of Mechanical and Manufacturing Engineering, School of Engineering, Trinity College Dublin, Dublin, Ireland. ${ }^{5}$ Department of Anatomy, Royal College of Surgeons in Ireland, Dublin, Ireland.

${ }^{6}$ Advanced Materials and Bioengineering Research Centre, Royal College of Surgeons in Ireland and Trinity College Dublin, Dublin, Ireland.

${ }^{7}$ Department of Mechanical Engineering, Colorado State University, Fort Collins, CO USA

${ }^{8}$ Centre for Medical Engineering Research, School of Mechanical and Manufacturing Engineering, Dublin City University, Dublin, Ireland

Hannah Marie Pauly

1376 Campus Delivery

School of Biomedical Engineering

Colorado State University

Fort Collins, CO 80523

Hmpauly1@gmail.com

Phone: (970) 297-4033

Binulal Nelson Sathy

Trinity Centre for Bioengineering

Trinity Biomedical Sciences Institute

Trinity College Dublin

152-160 Pearse St

Dublin 2

Ireland

Binulalns@gmail.com

Phone: +353-1-877864246

Dinorath Olvera

Trinity Centre for Bioengineering

Trinity Biomedical Sciences Institute

Trinity College Dublin

152-160 Pearse St

Dublin 2

Ireland

Dinorath.olvera@gmail.com

Phone: +353-1-877864246

Helen O McCarthy

School of Pharmacy

Queen's University of Belfast

Medical Biology Centre

97 Lisburn Road

Belfast

BT9 7BL

United Kingdom

H.mccarthy@qub.ac.uk 
Phone: +353-1-7005712

Daniel John Kelly

Trinity Centre for Bioengineering

Trinity Biomedical Sciences Institute

Trinity College Dublin

152-160 Pearse St

Dublin 2

Ireland

KELLYD9@tcd.ie

Phone: +353-1-8963947

Fax: +353-1-6795554

Ketul C Popat

1374 Campus Delivery

Department of Mechanical Engineering

Colorado State University

Fort Collins, CO 80523

Ketul.Popat@colostate.edu

Phone: (970)491-1468

Nicholas J Dunne

Centre for Medical Engineering Research

School of Mechanical and Manufacturing Engineering

Dublin City University

Stokes Building

Collins Avenue

Dublin 9

Ireland

Nicholas.dunne@dcu.ie

Phone: +353-1-7005712

Corresponding author:

Tammy Lynn Haut Donahue

1374 Campus Delivery

Department of Mechanical Engineering

Colorado State University

Fort Collins, CO 80523

Tammy.Donahue@ColoState.edu

Phone: (970) 491-1319

Fax: (970) 491-3827 


\begin{abstract}
The anterior cruciate ligament (ACL) of the knee is vital for proper joint function and is commonly ruptured during sports injuries or car accidents. Due to a lack of intrinsic healing capacity and drawbacks with allografts and autografts, there is a need for a tissue engineered ACL replacement. Our group has previously used aligned sheets of electrospun polycaprolactone nanofibers to develop solid cylindrical bundles of longitudinally aligned nanofibers. We have shown that these nanofiber bundles support cell proliferation and elongation and the hierarchical structure and material properties are similar to the native human ACL. It is possible to combine multiple nanofiber bundles to create a scaffold that attempts to mimic the macro-scale structure of the ACL. The goal of this work was to develop a hierarchical bioactive scaffold for ligament tissue engineering using connective tissue growth factor (CTGF) conjugated nanofiber bundles and evaluate the behavior of mesenchymal stem cells (MSCs) on these scaffolds in vitro and in vivo. CTGF was immobilized onto the surface of individual nanofiber bundles or scaffolds consisting of multiple nanofiber bundles. The conjugation efficiency and the release of conjugated CTGF was assessed using X-ray photoelectron spectroscopy, assays, and immunofluorescence staining. Scaffolds were seeded with MSCs and maintained in vitro for 7 days (individual nanofiber bundles), in vitro for 21 days (scaled-up scaffolds of 20 nanofiber bundles) or in vivo for 6 weeks (small scaffolds of 4 nanofiber bundles) and ligament specific tissue formation was assessed in comparison to non-CTGF conjugated control scaffolds. Results showed that CTGF conjugation encouraged cell proliferation and ligament specific tissue formation in vitro and in vivo. The results suggest that hierarchical electrospun nanofiber bundles conjugated with CTGF are a scalable and bioactive scaffold for ACL tissue engineering.
\end{abstract}




\section{Introduction}

The anterior cruciate ligament (ACL) is located in the knee and runs from the lateral posterior aspect of the femoral condyle to the medial anterior aspect of the tibial plateau ${ }^{1}$. The ACL is vital for joint stability and it functions primarily as a restraint to excessive translations of the tibia relative to the femur

${ }^{2,3}$. The ACL is primarily composed of collagen fibers arranged in a unique hierarchical structure and a lack of vascularization means that the ligament has a low intrinsic healing capacity ${ }^{4,5}$. It is estimated that up to 200,000 ACL injuries occur in the United States annually and the monetary burden of these injures and subsequent treatment is estimated to be $\$ 1$ billion each year ${ }^{6,7}$. Untreated tears of the ACL can cause knee pain, bone bruising, excessive joint laxity, and damage to the surrounding cartilage and menisci of the knee. Following an ACL injury, surgical replacement of the ACL with an autograft or allograft is typically attempted to restore function and stability to the injured knee. However drawbacks exist to both intervention techniques. Although allografts have been historically successful, morbidity at the site of tissue donation can result in pain, tendonitis and weakness ${ }^{8,9}$. Allograft tissue obtained from cadavers can also be used to replace the ACL however tissue supply is limited and disease transmission can be problematic $^{10}$. Thus there is interest in developing a tissue engineered replacement ACL that can overcome the limitations of traditional allografts and autografts.

The field of tissue engineering aims to combine scaffolds, cells, and signaling molecules to create a replacement for damaged biological tissue. Electrospinning is a versatile scaffold fabrication technique which uses biodegradable polymers to create a nanoscale structure that mimics the structure of the extracellular matrix ${ }^{11,12}$. Braided electrospun scaffolds have been shown to encourage cell proliferation and upregulation of tenogenic markers ${ }^{13,14}$ whereas laminated electrospun sheets have successfully encouraged cell infiltration ${ }^{15}$. However, both of these approaches have demonstrated inferior mechanical properties $^{12-14}$. A tissue engineered ACL that matches the hierarchical structure of the native ligament, has sufficient mechanical properties, and encourages the production of ligamentous tissue deposition in vitro and in vivo will likely improve the clinical treatment of ACL injury. 
Our group has previously developed a technique for modifying flat sheets of aligned electrospun polycaprolactone (PCL) nanofibers to create a more robust structure ${ }^{16}$. These cylindrical "nanofiber bundles" are approximately $500 \mu \mathrm{m}$ in diameter and are composed of nanofibers aligned along the longitudinal axis that mimic the native ACL structure. Nanofiber bundles are able to support short term cell growth and elongated cell morphology and have material properties that are comparable to that of the native human $A C L^{16-18}$. The smallest functional units of the ACL are collagen fibrils that range in size from $50-500 \mathrm{~nm}$ and are primarily oriented parallel to the longitudinal axis ${ }^{19,20}$. The individual PCL nanofibers that compose the electrospun sheets used in this study mimic this size scale ${ }^{16}$. Collagen fibrils of the ACL are organized into fibers and numerous collagen fibers are grouped into fascicles, approximately $100-500 \mu \mathrm{m}$ in size. The size of these collagen fascicles is similar to the size of one nanofiber bundles. In the native ACL, multiple collagen fascicles are grouped together to form the entire ligament. Thus, in an effort to mimic the hierarchical ACL structure, multiple nanofiber bundles were combined together to form a 3D scaffold for this study.

Growth factors are chemical signaling molecules that can be used to direct cell behavior. In the context of ligament tissue engineering it would be beneficial to utilize a growth factor that encourages cell proliferation as well as the deposition of extracellular matrix material characteristic of native ligaments ${ }^{21}$. Connective tissue growth factor (CTGF) is a 36-38 kDa heparin-binding protein characterized by multiple conserved cysteine-rich domains ${ }^{22}$. CTGF is involved in a variety of physiological processes including angiogenesis, embryo development, and would healing ${ }^{23}$. CTGF has been previously shown to encourage fibroblastic differentiation of stem cells, as evidenced by cell proliferation, gene expression changes, and increased matrix deposition ${ }^{22,24}$. The most common way to expose cells to growth factors is by introducing the growth factor to the cells in vitro in the cell growth media. The major drawback to this technique is that growth factors have a relatively short half-life in cell growth media and are rapidly inactivated, thus making it necessary to introduce fresh growth factor during each media change ${ }^{25}$. Additionally, sustained growth factor delivery in an in vivo setti ng is a challenge that needs to be addressed to facilitate the development of functionalized scaffolds. One strategy to address these 
challenges is to conjugate growth factors to the scaffold surface using a covalent chemical conjugation procedure $^{26}$. This surface modification-technique utilizes chemical bonds to immobilize the growth factors to exposed functional groups on a scaffold. Chemical conjugation of growth factors to a scaffold creates functionalized nanofiber scaffolds with tunable growth factor release and a prolonged influence on cell behavior ${ }^{25,26}$. Previously, electrospun scaffolds with chemically conjugated growth factors have been used to encourage neuronal differentiation, enhance wound healing, and stimulate osteogenic cellular $\operatorname{activity}^{27-29}$.

Previously, we have shown that individual PCL nanofiber bundles support short term in vitro cell growth and proliferation ${ }^{16}$, hence, this study is aimed at assessing long term cell behavior on a 3D scaffold with multiple nanofiber bundles and chemically conjugated CTGF, both in vitro and in vivo. It is hypothesized that the combination of the unique hierarchical structure and the presence of CTGF will induce the deposition of tissue that is characteristic of ligaments, primarily collagen type I and type III.

\section{Methods}

\subsection{Scaffold fabrication}

Flat sheets of aligned nanofibers were fabricated using an electrospinning technique as detailed previously ${ }^{16}$. A $10 \%$ w/v solution of polycaprolactone (PCL, $M_{w}=80,000$, Sigma 440744, St. Louis, MO) was prepared in a 3:1 v/v mixture of chloroform and methanol. The electrospinning solution was ejected horizontally from a glass syringe outfitted with an 18-gauge blunt tipped needle at a rate of $2 \mathrm{~mL} / \mathrm{hr}$ for $15 \mathrm{~min}$. To create sheets of aligned nanofibers (Figure 1A), the collector surface was a rotating aluminum drum located $10 \mathrm{~cm}$ from the needle tip and rotating with a linear velocity of approximately $12 \mathrm{~m} / \mathrm{s}$. These sheets were further used to create cylindrical bundles of nanofibers (Figure 1B) by excising a rectangular

portion of the sheet (approximately $5 \mathrm{~cm}$ x $175 \mathrm{~cm}$ ) and rolling it up tightly into a cylinder ${ }^{16}$. This resulted in nanofiber bundles that were approximately $0.5 \mathrm{~mm}$ in diameter and $175 \mathrm{~mm}$ length, with nanofibers aligned along the longitudinal axis. For further studies nanofiber bundles were cut to $20 \mathrm{~mm}$ in length. 


\section{$\underline{2.2 \text { Growth factor conjugation }}$}

CTGF (Sigma SRP4702, St. Louis, MO) was covalently bound to nanofiber bundles ( $n=3$ ) using a multi-step chemical conjugation technique (Figure 2A). First amines were added by incubating nanofiber bundles in a 5\% v/v aqueous solution of polyallylamine (Sigma 479136, St. Louis, MO) with simultaneous ultraviolet radiation for $25 \mathrm{~min}$. Next nanofiber bundles were incubated on a shaker plate in a solution $10 \%$ w/w solution of N-succinimidyl-3-maleimidoproprionate (TCI America SO427, Portland, OR) N,N-dimethylformamide (Sigma 227056, St. Louis, MO) for $1 \mathrm{hr}$ to add on maleimide terminals. In the final step, the nanofiber bundles were incubated in a $50 \mathrm{ng} / \mathrm{mL}$ solution of CTGF for $2 \mathrm{hr}$. Between subsequent steps the nanofiber bundles were rinsed in triplicate using deionized water $\left(\mathrm{dH}_{2} \mathrm{O}\right)$, incubated in distilled $\mathrm{dH}_{2} \mathrm{O}$ for $2 \mathrm{hr}$, and then rinsed an additional 3 times in $\mathrm{dH}_{2} \mathrm{O}$ to ensure all excess chemicals had been leached from the nanofibers.

CTGF conjugation efficiency was assessed using X-ray photoelectron spectroscopy (XPS, PE5800, Physical Electronics, Chanhassen, MN). XPS was conducted after each subsequent modification step to assess the surface composition of nitrogen, which is indicative of successful conjugation of each linker component as well as CTGF. Survey spectra were collected from 0 to $1100 \mathrm{eV}$ with pass energy of 187.85 and data for the percent elemental composition were calculated using the instrument specific software (PHI MultiPak, Physical Electronics, Chanhassen, MN).

Distribution of CTGF was assessed using immunofluorescence labeling. To immunofluorescently label the conjugated CTGF, nanofiber bundles were first rinsed with phosphate-buffered saline (PBS) and then incubated with 10\% donkey serum (Santa Cruz Biotechnology sc-2044, Santa Cruz, CA) in PBS for 20 min. After rinsing again in PBS, nanofiber bundles were incubated in the primary antibody against CTGF (goat, Santa Cruz Biotechnology sc-14939, Santa Cruz, CA) prepared in 1.5\% donkey serum at a concentration of $4 \mu \mathrm{g} / \mathrm{mL}$ in PBS for $1 \mathrm{hr}$. Nanofiber bundles were then rinsed in PBS in triplicate for 5 min each. Next, nanofiber bundles were incubated in the secondary antibody labeled with fluorescein isothiocyanate (FITC, donkey anti-goat, Santa Cruz Biotechnology sc-2024, Santa Cruz, CA) prepared in 
$1.5 \%$ donkey serum at a concentration of $4 \mu \mathrm{g} / \mathrm{mL}$ in PBS for $1 \mathrm{hr}$. Nanofiber bundles were rinsed again in PBS prior to imaging with a fluorescent microscope (Olympus IX70, Center Valley, PA).

An enzyme-linked immunosorbent assay (ELISA, PeproTech 900-m317, Rocky Hill, NJ) was used to characterize the amount of CTGF initially conjugated to each nanofiber bundle as well as the cumulative release of CTGF from nanofiber bundles over a 2 week time period. After the final conjugation step the CTGF solution was used to measure the amount of growth factor that remained unconjugated. Then nanofiber bundles with conjugated CTGF were incubated in $\mathrm{dH}_{2} \mathrm{O}$ at $37^{\circ} \mathrm{C}$ and $5 \%$ $\mathrm{CO}_{2} . \mathrm{DH}_{2} \mathrm{O}$ was collected from the nanofiber bundles at Days $1,2,5,7,10$, and 14 and the amount of CTGF in the solution was measured following the manufacturer instructions provided with the ELISA kit. This provided a cumulative measure of the percentage of conjugated CTGF that was released from the nanofiber bundles.

\section{$\underline{2.3 \text { Harvest of bone marrow-derived stem cells }}$}

Ovine bone marrow-derived mesenchymal stem cells (OBMSCs) for the in vitro experiments were aseptically harvested from the bone marrow of the femurs of 4 skeletally mature sheep that were euthanized for unrelated purposes. After disarticulating the leg, the muscle and fascia were removed and the femur was sawn open close to the femoral head. Bone marrow ( $\sim 5 \mathrm{~mL}$ from each femur) was removed from the medullary canal and transferred to a $50 \mathrm{~mL}$ tube containing cell growth media. The cell growth media consisted of Dulbecco's Modified Eagle Medium with 10\% fetal bovine serum (FBS), 1\% penicillin/streptomycin, and $2.5 \mu \mathrm{g} / \mathrm{mL}$ amphotericin B. Bone marrow was gently aspirated to break up tissue aggregates prior to centrifugation at $650 \mathrm{~g}$ for $5 \mathrm{~min}$. The separated fatty layer was discarded and the cell pellet was resuspended in $20 \mathrm{~mL}$ of cell growth media, triturated through a 16 gauge needle and filtered through a $40 \mu \mathrm{m}$ nylon cell strainer. Red blood cells were lysed using $4 \%$ acetic acid and mononuclear cells were counted using an automatic cell counter (Scepter 2.0, MilliporeSigma, Billerica, MA). Isolated OBMSC from the 4 animals were pooled, seeded at a density of 5,000 cells $/ \mathrm{cm}^{2}$ in $75 \mathrm{~cm}^{2}$ flasks and expanded until passage 4. Passage 4 OBMSCs were used for the in vitro experiments. 
Porcine bone marrow-derived mesenchymal stem cells (PBMSCs) for the in vivo experiments were isolated and expanded as previously described ${ }^{30}$. Briefly, mononuclear cells were isolated from the femora of 4 month old pigs as described above and seeded at a density of $5,000 \mathrm{cells} / \mathrm{cm}^{2}$ in $75 \mathrm{~cm}^{2}$ flasks. PBMSCs were maintained in cell growth media consisting of high glucose Dulbecco's modified Eagle's medium GlutaMAX, 10\% FBS, 1\% penicillin/streptomycin and $2.5 \mu \mathrm{g} / \mathrm{mL}$ amphotericin B and were expanded until passage 2. Passage 2 PBMSCs were used for the in vivo experiments.

\subsection{Short-term in vitro cell culture}

To assess the short-term effects of the conjugated CTGF on nanofiber bundles, OMBSCs were seeded on individual nanofiber bundles ( $n=6$, Figure 1B) both with and without conjugated CTGF. After CTGF conjugation samples were sterilized using $70 \%$ ethanol and exposure to ultra-violet light.

OBMSCs at passage 4 were seeded on nanofiber bundles at a concentration of approximately 200 cells/ $\mu \mathrm{L}$. Nanofiber bundles seeded with OBMSCs were maintained at $37^{\circ} \mathrm{C}$ and $5 \% \mathrm{CO}_{2}$ for 1 week and the cell growth media was changed every 2-3 days. Cell viability $(n=6)$ was measured after 1 , 4, and 7, days of culture using a CellTiter-Blue Cell Viability Assay (Promega G808A, Madison, WI) according to the manufacturer's instructions.

After 7 days in culture, cell adhesion was assessed by staining the cells on nanofiber bundles (n=3) with 5-Chloromethylfluorescein Diacetate (CMFDA, Life Technologies C2925, Carlsbad, CA), rhodamine-phalloidin (Cytoskeleton, Inc, Denver, CO) and 4',6-diamidino-2-phenylindole (DAPI, Life Technologies D1306, Carlbad, CA). The cytoplasm of live cells was stained green with CMFDA followed by fixation in $3.7 \%$ formaldehyde for $50 \mathrm{~min}$ and permeabilization with $1 \%$ Triton-X 100 for 3 min. After permeabilization rhodamine-phalloidin was used to stain the actin cytoskeleton of cells red and DAPI was used to stain the cell nuclei blue. Nanofiber bundles were imaged with a Zeiss Axioplan 2 fluorescence microscope. Additionally, scanning electron microscopy (SEM) was used to assess the morphology and spatial organization of cells on nanofiber bundles $(n=3)$ as previously described ${ }^{16}$. Briefly, cells were fixed for imaging in a solution of $3 \%$ glutaraldehyde, $0.1 \mathrm{M}$ sodium cacodylate, and 
0.1 M sucrose for $45 \mathrm{~min}$, then serially dehydrated and stored in a desiccator until imaged with an SEM (JSM-6500F, JOEL, Peabody, MA) operating at 10kV.

\subsection{Long-term in vitro cell culture}

A scaled-up scaffold $(n=12)$ composed of multiple nanofiber bundles was used to assess the long-term influence of conjugated CTGF on cells grown in vitro. This scaled-up scaffold was $\sim 3 \mathrm{~mm}$ in diameter and consisted of $\sim 20$ nanofiber bundles cut to $20 \mathrm{~mm}$ in length tied together at the ends with suture wire (Figure 1C). After fabrication scaffolds were either conjugated with CTGF (as previously described) or left unconjugated to serve as a control. OBMSCs at passage 4 were seeded on scaffolds at a concentration of approximately 600 cells $/ \mu \mathrm{L}$. Scaffolds were maintained in standard cell culture conditions for 21 days. Cell viability of scaffolds $(n=12)$ was measured at days $1,7,14$, and 21 using a CellTiter-Blue viability assay.

At the end of the 21 day culture period scaffolds were assessed using histological and immunohistochemical staining and biochemical assays. To assess the biochemical content scaffolds $(\mathrm{n}=8)$ were cut in half and rinsed in PBS and immediately frozen at $-80^{\circ} \mathrm{C}$. After thawing, scaffolds were digested with papain $(125 \mathrm{ug} / \mathrm{mL})$ in $0.1 \mathrm{M}$ sodium acetate, $5 \mathrm{mM}$ L-cysteine-HCL, and $0.05 \mathrm{M}$ ethylenediaminetetraacetic acid (EDTA) under constant rotation for $18 \mathrm{hr}$. Immediately after the papain digestion DNA content was analyzed using a PicoGreen DNA assay (Thermo Fisher Scientific P11496, Waltham, MA) . Total collagen content was determined by measuring the hydroxyproline content, after acidic hydrolysis of samples $\left(110^{\circ} \mathrm{C}\right.$ for $\left.18 \mathrm{hr}\right)$ in concentrated hydrochloric acid $(38 \%)^{31}$. Samples were assayed using a chloramine-T assay assuming a hydroxyproline-to-collagen ratio of 1:7.69 ${ }^{32}$. The amount of sulphated glycosaminoglycans (sGAG) was quantified using a dimethylmethylene blue dye-binding (DMMB) assay as previously reported ${ }^{31}$.

Additional halves of scaffolds $(n=8)$ were used for histology and immunohistochemistry. Scaffolds were fixed for $24 \mathrm{hr}$ in $10 \%$ formalin, infiltrated with a graded series of sucrose solutions, embedded in optimum cutting temperature medium (Tissue-Tek, Sakura Finetek, Torrance, CA), and flash frozen in liquid nitrogen. Embedded scaffolds were cryosectioned axially through the center to 
obtain $10 \mu \mathrm{m}$ slices which were fixed to microscope slides. Sections were stained with picrosirius red to assess collagen deposition, $1 \%$ alizarin red to assess calcium accumulation, and hematoxylin and safraninO/fast green to assess sulfated glycosaminoglycan content. Stained sections from day 1 were included as controls. Immunohistochemical analysis of collagen types I and III was performed using a standard immunohistochemical technique to evaluate the collagen types deposited by cells. Briefly, sections were pre-treated with pronase (Sigma-Aldrich PRON-RO, St Louis, MO) in a humidified environment at $37^{\circ} \mathrm{C}$ for $5 \mathrm{~min}$ for antigen retrieval. Then, sections were blocked with goat-serum (Sigma-Aldrich G9023, St Louis, MO) for $1 \mathrm{hr}$ and incubated with primary antibodies specific to collagens type I and III overnight at $4{ }^{\circ} \mathrm{C}$. For in vivo samples primary antibodies, anti-collagen I antibody [Col-1] (mouse monoclonal ab90395) or anti-collagen III antibody [1E7-D7/Col-3] (mouse monoclonal ab23445) were used. For in vitro samples primary antibodies, anti-collagen I antibody [5D8-G9] (mouse monoclonal ab23446) or anti-collagen III antibody (rabbit polyclonal ab7778) were used (all Abcam, Cambridge, UK). After overnight incubation, sections were washed in PBS and incubated with the secondary antibody, goat anti-mouse IgG (B7151, Sigma) or goat anti-rabbit IgG (20019, Biotium) for 1 h. Color was developed using the Vectastain $\mathrm{ABC}$ reagent kit (Vectastain ABC kit, Vector Laboratories, UK) followed by exposure to peroxidase DAB peroxidase substrate (DAB Peroxidase (HRP) Substrate Kit, Vector Laboratories, UK ). Reaction was stopped when color was visible and sections were washed, dehydrated through an alcohol gradient and mounted using Vectashield (Vector Laboratories, UK).

\section{$\underline{2.6 \text { In vivo subcutaneous implantation }}$}

A smaller scaffold $(n=8)$ consisting of 4 nanofiber bundles secured together at one end was implanted subcutaneously into the back of a nude mouse (Figure 1D). The size of the scaffold was adjusted so that it could be used with the nude mouse. CTGF was conjugated to the small scaffold surface as described above and control scaffolds were also included with no growth factors. PBMSCs at passage 2 were seeded on small scaffolds at a concentration of approximately 2000 cells $/ \mu \mathrm{L}$

All animal experiments were approved by the Ethics Committee of Trinity College Dublin (Republic of Ireland) and the Irish Medicines Board (IMB, Republic of Ireland). Control and CTGF 
conjugated small scaffolds ( $\mathrm{n}=8$ per group) were implanted into the subcutaneous space of Balb/C nude mice (Harlan, UK) using a previously described surgical procedure ${ }^{33}$ Briefly, 2 subcutaneous pockets were created on both flanks of the mice under aseptic conditions through an incision made on the dorsal side of the mouse. Then, one small scaffold was implanted in each subcutaneous pocket $(2$ small scaffolds per animal). All small scaffolds were harvested at 6 weeks of in vivo implantation. Small scaffolds were analyzed for gross appearance and collagenous tissue formation by picrosirius red histology and immunohistochemistry, as described above.

\subsection{Statistics}

Data is presented as mean \pm standard error of the mean. Data analysis was performed using Minitab (Minitab, Inc., State College, PA). For all quantitative measures, comparisons were performed using a two-way analysis of variance (ANOVA) with Tukey's post-hoc test. Differences corresponding to a p-value of less than 0.05 were considered to be statistically significant.

\section{Results}

\subsection{Growth factor conjugation}

The covalent chemical conjugation of CTGF to electrospun nanofibers was verified using XPS analysis after modification of the nanofiber bundles with the linker molecules and CTGF. Initially since unmodified PCL consists only of carbon and oxygen atoms there is no nitrogen present. After modifying with polyallylamine to add amines groups on the surface of the nanofiber bundles, the surface elemental nitrogen composition was $1.5 \%$. The addition of maleimides terminals using $\mathrm{N}$-succinimidyl-3maleimidoproprionate resulted in a $0.8 \%$ increase in the nitrogen composition of the surface. Finally after adding the growth factor CTGF, which consists of multiple conserved cysteine rich domains, the final nitrogen composition was $3.5 \%$. The increase in nitrogen could be seen visually by inspecting the nitrogen peak of the XPS survey before and after CTGF conjugation (Figure 2B). This increase in elemental nitrogen percentage after each subsequent modification step indicates successful conjugation. 
Immunofluorescence labeling demonstrated that the conjugated CTGF was distributed uniformly across the surface of nanofiber bundles, as intended (Figure 2C). A CTGF ELISA was used to determine how much CTGF did not conjugate on to the nanofiber surfaces, as a quantitative measure of conjugation efficiency. During the conjugation procedure each sample was exposed to $10 \mathrm{ng}$ of $\mathrm{CTGF}$ in $\mathrm{dH}_{2} \mathrm{O}$. After the $2 \mathrm{hr}$ incubation period, an average of only $0.466( \pm 0.028)$ ng of CTGF remained in the $\mathrm{dH}_{2} \mathrm{O}$ (Figure 2D). This demonstrates that $95 \%$ of the CTGF was successfully conjugated to the nanofiber bundles. The same ELISA was also used to assess how much of CTGF was released from nanofiber bundles over a 2 week time period. The results showed that over the 2 week time period less the $6 \%$ of conjugated CTGF was released from the samples (Figure 2E). In the first 5 days there was a slight burst release where $~ 5 \%$ of CTGF was released however, in the following 9 days only an additional $1 \%$ of the CTGF was released $\underline{\text { 3.2 Short-term in vitro cell culture }}$

The CellTiter-Blue metabolic assay revealed that cells attached and remained viable on individual nanofiber bundles both with and without conjugated CTGF for up to 7 days in culture (Figure 3A). There were no differences in metabolic activity level of the cells on nanofiber bundles with or without CTGF, and the metabolic activity level of cells on all nanofiber bundles remained relatively constant over time. SEM images showed that cells adhered, spread, and colonized on all nanofiber bundles (Figure 3B, 3D). Additionally cells appeared to have adopted an elongated morphology along the direction of the nanofibers (along the longitudinal axis of the nanofiber bundle) for all nanofiber bundles. Similarly, after 7 days of culture, fluorescence microscopy images showed elongated cells covering the surfaces of all nanofiber bundles (Figure 3C, 3E).

\subsection{Long-term in vitro cell culture}

The CellTiter-Blue assay revealed significant differences in the metabolic activity of cells on control and CTGF scaffolds consisting of $\sim 20$ nanofiber bundles. At each time point investigated the metabolic activity of cells on CTGF scaffolds was significantly higher than the metabolic activity of cells grown on control scaffolds (Figure 4A). Additionally the metabolic activity of cells on scaffolds conjugated with CTGF was increased significantly from Day 1 to Day $21(p=0.002)$. In contrast the 
metabolic activity of cells on control scaffolds did not significantly change from Day 1 to Day 21 $(p=0.142)$. Similarly, the PicoGreen Assay to measure DNA content of the scaffolds showed that after 1 day in culture the DNA content of cells on CTGF scaffolds was significantly higher DNA content of control scaffolds ( $p=0.01$, Figure 4B). By Day 21 the DNA content of cells on CTGF scaffolds had increased significantly from Day $1(p<0.0001)$ while the DNA content of control scaffolds did not significantly change $(p=0.234)$.

Biochemical analysis revealed that by Day 21 the amount of accumulated collagen (normalized to $\mu \mathrm{g}$ of DNA) in CTGF conjugated scaffolds was significantly higher than the collagen accumulated in control scaffolds ( $p=0.031$, Figure $4 \mathrm{C}$ ). The DMMB assay to determine sGAG accumulation within the scaffolds showed that by Day 21 of culture the sGAG content (normalized to $\mu$ g of DNA) of CTGF scaffolds was significantly lower than the sGAG content of control scaffolds ( $p=0.0008$, Figure 4D).

Histology performed using a picrosirius red stain after 21 days of culture showed that there was notable collagen deposition by cells grown on CTGF scaffolds compared to control scaffolds (Figure 5A, 5B). The collagen was deposited primarily around nanofiber bundles that were on the exterior of the scaffold, which some deposition reaching towards the interior. There was no notable deposition of glycosaminoglycans (red/pink stain) or calcium (bright red), based on safraninO/fast green and alizarin red stains respectively, on either control or CTGF scaffolds (Figure 5C-F). Immunohistochemical analysis showed that there was no apparent labeling of collagen on control scaffolds (Figure 5G, 5I). The collagen deposited on the CTGF scaffolds stained positive for collagen type I and type III (Figure 5H, 5J).

\section{$\underline{3.4 \text { In vivo subcutaneous implantation }}$}

CTGF conjugated scaffolds implanted in vivo showed more homogenous collagen coverage around the nanofiber bundles whereas the control in vivo small scaffolds showed uneven covering of collagen with different staining intensity in some places around the nanofiber bundles (Figure 6A, 6B). The collagen type I and III immunohistochemistry showed uniform staining around the CTGF conjugated nanofiber bundles indicating the even distribution of ligament specific tissue in presence of CTGF (Figure $6 \mathrm{D}, 6 \mathrm{~F})$. The periphery of control nanofiber bundles stained intensely for collagen type I staining and 
relatively weakly for collagen type III (Figure 6C, 6E). This could indicate that non-specific fibrous tissue is forming on the unconjugated nanofiber bundles.

\section{Discussion}

The key requirements for a tissue engineered ligament scaffold have been well described previously ${ }^{21,34}$. The scaffold must be made from a biocompatible material and closely match the structural and mechanical properties of the native tissue. The scaffolds investigated in this work represent a hierarchal construct that mimics the structure of the native human ACL using sheets of aligned nanofibers prepared using a standard electrospinning technique. By rolling rectangular sections of the electrospun nanofiber sheets to create nanofiber bundles it creates a robust structure which supports cell growth and proliferation, cell elongation, and has material properties that are comparable to the properties of the native human ACL ${ }^{16-18}$. Other have attempted to use stacked PCL sheets for ACL replacement and while transient immune responses were noted ${ }^{15}$, they subsided and the potential for heparin mediated growth factor release was noted, as a technique for further encouraging de novo extracellular matrix deposition $^{35,36}$. Similarly, Bosworth et al. reported that tightly wound PCL yarn could be utilized as a substrate for tendon fibroblast adhesion and proliferation ${ }^{37}$. Modifications to traditional PCL have also been investigated as materials for ligament scaffolds, particularly ultra-high molecular weight PCL (UHMWPCL) and PCL with the addition of L-lactic acid (PCLC) with promising results ${ }^{38,39}$. In the current study, a scaffold composed of multiple nanofiber bundles was created to better mimic the hierarchal structure of the collagen in the native ACL on macro, micro, and nano size scales, and provide space for matrix deposition between and around the nanofiber bundles.

In addition to scaffold material, it is also important to consider the structure of the scaffold. The native ACL possess a complex hierarchical structure with collagen fibrils primarily oriented along the longitudinal axis of the ligament ${ }^{20}$, and then organized into fascicles ${ }^{4}$. The primarily parallel orientation is thought to lend tensile strength and stability to the ligament ${ }^{5}$. The scaffold presented in this work was fabricated to attempt to mimic this hierarchical structure. The electrospun PCL nanofibers which mimic 
the collagen fibrils are tightly rolled upon themselves to create nanofiber bundles which mimic the collagen fascicles. Previous work showed that these individual nanofiber bundles promoted cell adhesion and proliferation ${ }^{16}$. In order to provide a suitable ligament replacement a scaffold must also be able to withstand the repeated tensile loading experienced by native ligaments. Previous mechanical testing revealed that when pulled to failure at a physiologically relevant strain rate the modulus, yield stress, and yield stress and yield strain of nanofiber bundles mimic the material properties of the native human ACL, suggesting this scaffold has the potential to meet the mechanical demands of the ligament ${ }^{16}$. To better mimic the overall ACL structure multiple nanofiber bundles were grouped together by tying the ends together, which allows the nanofiber bundles to lie parallel to one another. Although the native collagen fascicles of ACL are not perfectly parallel to one another, particularly when comparing fascicles in the in the anteromedial and posterolateral bundles of the ligament, this scaffold structure represents a first step at mimicking the hierarchical ACL structure. Other groups have attempted to recreate the hierarchical ACL structure with various techniques. Two popular techniques are utilizing braided or twisted strands of material to increase stiffness and tensile strength, however encouraging cell infiltration can be challenging ${ }^{13,39-42}$. A recent study comparing braided electrospun PCL sheets to stacked electrospun PCL sheets showed that the braided scaffolds possessed improved tensile strength which better mimicked the native ACL, however cell infiltration and proliferation was superior in stacked constructs ${ }^{43}$.

In order to encourage cell adhesion and proliferation as well as the deposition of native ligament ECM by the cells, we investigated the chemical conjugation of growth factors on the surface of the scaffold. The chemical conjugation technique used in this study resulted in a 95\% conjugation efficiency, which suggests a secure covalent bond between the PCL, linker molecules, and the CTGF. After an initial burst release, over a two week time period only $\sim 6 \%$ of the conjugated CTGF was released from the scaffold. Previous studies using similar techniques to covalently immobilize growth factors on scaffold surfaces have showed similar release profiles ${ }^{28,44}$. The initial burst release of CTGF into the surrounding media could be due to the release of some unconjugated CTGF that was physically trapped within the porous network of nanofibers and required multiple days to diffuse out into the surrounding solution. The 
CTGF that remains covalently conjugated to the nanofiber bundles is thought to influence cells that adhere to the scaffold via juxtacrine signal transduction ${ }^{45}$. When cells are in contact with the conjugated growth factors, signal transduction pathways are initiated which lead to cellular adaptation and upregulation of collagen I and III production ${ }^{29}$.

After one week of in vitro culture there was no difference in cell metabolic activity on nanofiber bundles with and without conjugated with CTGF. Thus, for early time points the presence of the CTGF likely has no impact on cell proliferation and viability. Other groups that have utilized growth factors for ligament tissue engineering have found differences in cell proliferation as early as 7 days in vitro.

Notably, after 1 week of culture Sahoo et al. demonstrated significantly higher viability when cells were grown on knitted silk scaffolds that had been with electrospun nanofibers made of a PLGA/basic fibroblastic growth factor (bFGF) blend, compared to the same scaffolds without $\mathrm{bFGF}^{46}$. However this response could be due to the release profile of the bFGF, since nearly $60 \%$ was released into the surrounding media in the first 7 days ${ }^{47}$. Similarly, Hankemeier et al. showed that after 7 days of culture higher doses of bFGF results in a higher density of cells ${ }^{48}$. On our scaffolds, no apparent differences in cell coverage on nanofiber bundle surfaces were noted, and cells became elongated along the long-axis of the nanofiber bundle regardless of CTGF presence. This is somewhat surprising since previous studies have shown that the presence of CTGF induces a more elongated cell shape ${ }^{22,24}$ however it could be that the aligned PCL nanofibers present in our scaffold encourage cell elongation regardless of growth factor presence. The shape and morphology of cells, as dictated by the structure of the scaffold, influence cell migration and communication as well as cellular differentiation ${ }^{49}$. In the context of ligament tissue engineering, an elongated spindle-shaped cell morphology mimics the structure of native fibroblasts and is thought to be important for fibroblastic cellular differentiation ${ }^{50,51}$.

In contrast to the cell proliferation seen on individual nanofiber bundles, when scaffolds consisting of $\sim 20$ nanofiber bundles were cultured in vitro, there were significant differences in cell proliferation in the presence of conjugated CTGF. As early as 1 day after cell seeding, DNA content and cellular metabolic activity on scaffolds with conjugated CTGF were both nearly double that of control 
scaffolds. This suggests that initially the cells had a greater affinity for attaching to scaffolds with conjugated CTGF. However, on individual nanofiber bundles, cells adhered at equal levels regardless of CTGF presence. This could be due to the differences in the macro-scale architecture of the scaffold. Since the 3D scaffold consisted of multiple nanofiber bundles, each conjugated with CTGF, the larger area of exposed surface area for growth factor could encourage more cell attachment. It is difficult to compare our initial cell attachment results to those of other groups, since typically cell behavior is not investigated after only one day of in vitro culture. However in the future it will be necessary to better understand what chemical and structural properties of scaffolds influence initial cell attachment. The trend for higher DNA content and higher metabolic activity on CTGF scaffolds persisted through day 21 of in vitro culture. Additionally after 21 days of in vitro culture the cell metabolic activity and the DNA content on CTGF conjugated scaffolds was higher than it had been at day 1, suggesting robust cellular proliferation throughout the 3 week culture period. These results closely match those of other research groups who have assessed the proliferation of cells both in the presence of other ligament-related growth factors $^{35,46,48,52}$, and specifically in the presence of $\mathrm{CTGF}^{22,24}$.

In addition to influencing cell adhesion and proliferation, conjugated CTGF had an effect on the matrix material deposited by cells in vitro. Collagen is the predominant extracellular matrix material in ligaments and its presence is crucial for repair and regeneration of fibrous tissues ${ }^{53}$. Robust deposition of collagen is vital for a successful tissue engineered ligament so that as the scaffold gradually degrades, de novo tissue remains in its place. Cells on all scaffolds deposited collagen around the nanofiber bundles. The collagen deposition was mostly observed around the outside of the scaffold where it bridged the gaps between nanofiber bundles, however some collagen deposition was also present toward the interior of the scaffold. Similarly, Copper et al. implanted braided scaffolds composed of PLLA fibers in a rabbit ACL reconstruction model and noted robust deposition of cells and dense connective tissue surrounding all polymer fibers ${ }^{54}$. The 21 day in vitro culture period investigated in the present study was chosen based on measureable changes in collagen deposition between the groups of control and CTGF conjugated scaffolds. Additionally, other research groups who have investigated the effects of growth factors on 
nanofibrous scaffolds in vitro have used similar 3 week time points to investigate cell behavior ${ }^{13,24,46,52,55}$. In order to further encourage more robust deposition of collagen around and between the individual nanofiber bundles that compose the 3D scaffold it may be necessary to incorporate mechanical stimulation. Previous groups have shown that compared to static culture, tensile loading of polymer scaffolds during culture encourages more cellular deposition of highly aligned collagen fibers similar to those observed in ligaments ${ }^{56,57}$. Additionally, nano-scale alterations such as modifying the nanofibers to have a crimp-like microarchitecture may encourage ECM deposition and cell differentiation ${ }^{58,59}$. On the scaffolds presented in this study, collagen deposition was notably more pronounced on scaffolds with conjugated CTGF. Quantitatively, the measured amount of accumulated collagen (normalized for DNA content) was nearly 4 times higher on CTGF scaffolds compared to control scaffolds. CTGF presence has been previously shown to stimulate the robust production of collagen in vitro in both fibroblasts and mesenchymal stem cells based on assessment of mRNA levels and histological staining of tissues ${ }^{22,23}$. Ideally, the extracellular matrix produced by cells on a tissue engineered ligament should mimic the structure and biological function of the native ligament ECM as much as possible. Thus, when considering collagen deposition on a scaffold it is important to not only note the presence of collagen, but also the specific types of collagen. Collagen type I is the major collagen found in ligaments however collagen type III is also present ${ }^{4}$. Type III collagen is found throughout the ACL as a component of reticular fibers, a loose connective tissue that divide type I collagen bundles. Immunohistochemistry after 3 weeks in vitro showed that on scaffolds with conjugated CTGF the collagen that was deposited on the surface of the nanofiber bundles was primarily types I and III. In contrast, on the control scaffolds there was essentially no evidence of either collagen I or III staining. There has been some evidence to suggest that the levels of different types of collagen fluctuate during tendon development, where type III collagen is deposited initially and later replaced by type I collagen ${ }^{60}$. Similarly during the connective tissue healing process some groups have found an initial increase in type III collagen followed by additional type I collagen deposition ${ }^{61,62}$. Thus in future work it may be beneficial to quantify the types of collagen present to assess early recapitulation of the ligament development and healing process. Additionally notable 
deposition of collagen types I and III could suggest that the OBMSCs seeded on the scaffolds are beginning to differentiate into fibroblasts, cells characteristic of ligaments ${ }^{63,64}$. In future studies, gene expression changes will be investigated to further confirm cell differentiation.

There was no apparent deposition of calcium or sGAG on any scaffolds. The absence of calcium is appropriate since no calcium is present in native ligaments. By Day 21 the amount of sGAG accumulated on CTGF scaffolds was lower than the accumulated sGAG on control scaffolds. Because high sGAG production is typically associated with chondrogenic differentiation, less accumulation of sGAG further suggests that the OBMSCs seeded on the scaffolds conjugated with CTGF may be differentiating into fibroblasts ${ }^{65}$. The ACL does have a small amount of sGAG, about $9 \%$ of the weight of the dry tissue, which is thought to be important for the viscoelastic mechanical behavior ${ }^{4,66}$. In future studies it may be necessary to optimize the amount of CTGF present in order to regulate the spatial deposition of both sGAG and collagen.

In an effort to better understand the in vivo response of cells to the scaffold and conjugated growth factor at a long time point, a nude mouse model was utilized. The smaller size of the scaffolds implanted in vivo permitted the assessment of matrix deposition between nanofiber bundles while conserving space and resources. The 6 week implantation period was chosen in order to be an extension of the in vitro time point and to get an initial indication of longer term in vivo cell behavior. After 6 weeks of in vivo implantation, collagen deposition was observed around all fibers of the scaffolds. The stacked electrospun PCL scaffold implanted in vitro by Petrigliano et al. in a rat model of ACL reconstruction showed that cells did not start producing a collagen matrix until 6 weeks after implantation, and this collagen matrix matured through week $12^{15}$. Thus is it possible that the collagen observed on our scaffolds in vitro would continue to increase and mature with additional implantation time. For the scaffolds presented in this study, in vivo collagen deposition appeared similar for both control and CTGF scaffolds. Differences in the influence of CTGF on collagen deposition in vitro and in vivo could be due to the longer time point, the presence of host cells in vivo, or the influence of other in vivo environmental cues. Additionally, the collagen deposited on all scaffolds (control and CTGF conjugated) in vivo was 
identified as collagen types I and III. This is a slightly different from what was observed in vitro, where types I and III collagen were only identified on CTGF conjugated scaffolds. This difference could be based on culture times, and potentially with longer in vitro culture times collagen I and III accumulation would increase on control scaffolds, similar to what was seen in vivo. Similarly, a knitted silk scaffold implanted in a porcine ACL reconstruction model was found to support robust deposition of collagen I and III and tenasin-C in vivo ${ }^{67}$. One limitation of the in vivo results presented is a lack of quantification of collagen content of the scaffolds. Non-specific fibrous tissue that can form around scaffolds during subcutaneous implantation can interfere with the hydroxyproline assay which is commonly used to measure collagen content. Thus we chose to only utilize histology and immunohistochemical staining for the specific types of collagen found in ligaments. An additional limitation of the in vivo work is that in the in vivo environment it is challenging to separate the activities of the cells seeded on the scaffold prior to implantation from host cells found within the animal. For example Spalazzi et al implanted a triphasic polymer scaffold subcutaneously in an athymic rat and found thorough host cell infiltration which led to

the deposition of de novo tissue within the scaffold ${ }^{68,69}$. Future studies will explore implanting cell-free and cell-laden scaffolds in more challenging orthotopic locations.

Overall the results of this study have demonstrated that it is possible to utilize CTGF conjugation and novel biofabrication strategies to create a bioactive scaffold that mimics the hierarchal structure of the native ACL. During in vitro and in vivo culture the presence of CTGF encourages cell adhesion, proliferation, and robust deposition of collagen which is specific for ligament tissue. This suggests that the presence of CTGF may be influencing the seeded stem cells to differentiate into ligamentous cells. Therefore the scaffold developed in this study, in combination with CTGF and cells, could serve as a tissue engineered ACL replacement.

\section{Acknowledgements}

This material is based upon work supported by the National Science Foundation under Grant No. DMR 1306741 and through the US-Ireland R\&D Partnership Programme (USI 004, SFI-12/US/I2489). The 
authors would like to acknowledge Jeremiah Easley and Kim Lebsock for their assistance in obtaining ovine femurs. 


\section{References}

1. Dye SF, Cannon WD: Anatomy and biomechanics of the anterior cruciate ligament. Clin Sports Med 7: 715, 1988.

2. Girgis FG, Marshall JL, Monajem A: The cruciate ligaments of the knee joint. Anatomical, functional and experimental analysis. Clin Orthop Relat Res: 216, 1975.

3. Woo SL, Hildebrand K, Watanabe N, Fenwick JA, Papageorgiou CD, Wang JH: Tissue engineering of ligament and tendon healing. Clin Orthop Relat Res: S312, 1999.

4. Duthon VB, Barea C, Abrassart S, Fasel JH, Fritschy D, Ménétrey J: Anatomy of the anterior cruciate ligament. Knee Surg Sports Traumatol Arthrosc 14: 204, 2006.

5. Arnoczky SP: Anatomy of the anterior cruciate ligament. Clin Orthop Relat Res: 19, 1983.

6. Giugliano DN, Solomon JL: ACL Tears in Female Athletes. Phys Med Rehabil Clin N Am 18: 417, 2007.

7. Prodromos CC, Han Y, Rogowski J, Joyce B, Shi K: A Meta-analysis of the Incidence of Anterior Cruciate Ligament Tears as a Function of Gender, Sport, and a Knee Injury-Reduction Regimen. Arthrosc - J Arthrosc Relat Surg 23: 1320, 2007.

8. Buss DD, Warren RF, Wickiewicz TL, Galinat BJ, Panariello R: Arthroscopically assisted reconstruction of the anterior cruciate ligament with use of autogenous patellar-ligament grafts. Results after twenty-four to forty-two months. J Bone Joint Surg Am 75: 1346, 1993.

9. Langan P, Fontanetta AP: Rupture of the patellar tendon after use of its central third. Orthop Rev 16: 317, 1987.

10. Simonds RJ, Holmberg SD, Hurwitz RL, Coleman TR, Bottenfield S, Conley LJ, Kohlenberg SH, Castro KG, Dahan BA, Schable CA: Transmission of human immunodeficiency virus type 1 from a seronegative organ and tissue donor. N Engl J Med 326: 726, 1992.

11. Pedicini A, Farris RJ: Mechanical behavior of electrospun polyurethane. Polymer (Guildf) 44: 6857, 2003.

12. Li D, Xia Y: Electrospinning of Nanofibers: Reinventing the Wheel? Adv Mater 16: 1151, 2004.

13. Barber JG, Handorf AM, Allee TJ, Li W-J: Braided nanofibrous scaffold for tendon and ligament tissue engineering. Tissue Eng Part A 19: 1265, 2013.

14. Freeman JW, Woods MD, Laurencin CT: Tissue engineering of the anterior cruciate ligament using a braid-twist scaffold design. J Biomech 40: 2029, 2007.

15. Petrigliano FA, Arom GA, Nazemi AN, Yeranosian MG, Wu BM, McAllister DR: In Vivo Evaluation of Electrospun Polycaprolactone Graft for Anterior Cruciate Ligament Engineering. Tissue Eng Part A 21: 1228, 2015. 
16. Pauly HM, Kelly DJ, Popat KC, Trujillo NA, Dunne NJ, Mccarthy HO, Donahue TLH: Mechanical Properties and Cellular Response of Novel Electrospun Nanofibers for Ligament Tissue Engineering : Effects of Orientation and Geometry. J Mech Behav Biomed Mater 61: 258, 2016.

17. Skelley NW, Castile RM, Cannon PC, Weber CI, Brophy RH, Lake SP: Regional Variation in the Mechanical and Microstructural Properties of the Human Anterior Cruciate Ligament. Am J Sports Med: 2892, 2016.

18. Paschos NK, Gartzonikas D, Barkoula NM, Moraiti C, Paipetis A, Matikas TE, Georgoulis AD: Cadaveric Study of Anterior Cruciate Ligament Failure Patterns Under Uniaxial Tension Along the Ligament. Arthrosc - J Arthrosc Relat Surg 26: 957, 2010.

19. Kastelic J, Galeski A, Baer E: The multicomposite structure of tendon. Connect Tissue Res 6: 11, 1978.

20. Danylchuk KD, Finlay JB, Krcek JP: Microstructural organization of human and bovine cruciate ligaments. Clin Orthop Relat Res 131: 294, 1978.

21. Petrigliano FA, McAllister DR, Wu BM: Tissue engineering for anterior cruciate ligament reconstruction: a review of current strategies. Arthroscopy 22: 441, 2006.

22. Lee CH, Shah B, Moioli EK, Mao JJ: CTGF directs fibroblast differentiation from human mesenchymal stem/stromal cells and defines connective tissue healing in a rodent injury model. $\mathrm{J}$ Clin Invest 120: 3340, 2010.

23. Moussad EDA., Brigstock DR: Connective Tissue Growth Factor: What's in a Name? Mol Genet Metab 71: 276, 2000.

24. Tong Z, Sant S, Khademhosseini A, Jia X: Controlling the Fibroblastic Differentiation of Mesenchymal Stem Cells Via the Combination of Fibrous Scaffolds and Connective Tissue Growth Factor. Tissue Eng Part A 17: 2773, 2011.

25. Lee K, Silva EA, Mooney DJ: Growth factor delivery-based tissue engineering: general approaches and a review of recent developments. J R Soc Interface 8: 153, 2011.

26. Yoo HS, Kim TG, Park TG: Surface-functionalized electrospun nanofibers for tissue engineering and drug delivery. Adv Drug Deliv Rev 61: 1033, 2009.

27. Choi JS, Leong KW, Yoo HS: In vivo wound healing of diabetic ulcers using electrospun nanofibers immobilized with human epidermal growth factor (EGF). Biomaterials 29: 587, 2008.

28. Mottaghitalab F, Farokhi M, Mottaghitalab V, Ziabari M, Divsalar A, Shokrgozar MA: Enhancement of neural cell lines proliferation using nano-structured chitosan/poly(vinyl alcohol) scaffolds conjugated with nerve growth factor. Carbohydr Polym 86: 526, 2011.

29. Zhang H, Migneco F, Lin C-Y, Hollister SJ: Chemically-conjugated bone morphogenetic protein2 on three-dimensional polycaprolactone scaffolds stimulates osteogenic activity in bone marrow stromal cells. Tissue Eng Part A 16: 3441, 2010. 
30. Thorpe SD, Nagel T, Carroll SF, Kelly DJ: Modulating Gradients in Regulatory Signals within Mesenchymal Stem Cell Seeded Hydrogels: A Novel Strategy to Engineer Zonal Articular Cartilage. PLoS One 8: 2013.

31. Guillaume O, Daly A, Lennon K, Gansau J, Buckley SF, Buckley CT: Shape-memory porous alginate scaffolds for regeneration of the annulus fibrosus: effect of TGF- $\beta 3$ supplementation and oxygen culture conditions. Acta Biomater 10: 1985, 2014.

32. Ignat'eva NY, Danilov NA., Averkiev S V., Obrezkova MV., Lunin VV., Sobol EN: Determination of hydroxyproline in tissues and the evaluation of the collagen content of the tissues. J Anal Chem 62: 51, 2007.

33. Vinardell T, Sheehy EJ, Buckley CT, Kelly DJ: A comparison of the functionality and in vivo phenotypic stability of cartilaginous tissues engineered from different stem cell sources. Tissue Eng Part A 18: 1161, 2012.

34. Leong NL, Petrigliano FA, McAllister DR: Current tissue engineering strategies in anterior cruciate ligament reconstruction. J Biomed Mater Res A 102: 1614, 2014.

35. Leong NL, Kabir N, Arshi A, Nazemi A, Wu B, Petrigliano FA, McAllister DR: Evaluation of polycaprolactone scaffold with basic fibroblast growth factor and fibroblasts in an athymic rat model for anterior cruciate ligament reconstruction. Tissue Eng Part A 21: 1859, 2015.

36. Leong NL, Arshi A, Kabir N, Nazemi A, Petrigliano FA., Wu BM, McAllister DR: In vitro and in vivo evaluation of heparin mediated growth factor release from tissue-engineered constructs for anterior cruciate ligament reconstruction. J Orthop Res 33: 229, 2015.

37. Bosworth L a, Alam N, Wong JK, Downes S: Investigation of 2D and 3D electrospun scaffolds intended for tendon repair. J Mater Sci Mater Med 24: 1605, 2013.

38. Leong NL, Kabir N, Arshi A, Nazemi A, Jiang J, Wu BM, Petrigliano FA., McAllister DR: Use of ultra-high molecular weight polycaprolactone scaffolds for ACL reconstruction. J Orthop Res 34: 828, 2016.

39. Laurent C, Ganghoffer J, Babin J: Morphological characterization of a novel scaffold for anterior cruciate ligament tissue engineering. J Biomecahnics 133: 011006, 2011.

40. Chung EJ, Sugimoto MJ, Koh JL, Ameer GA: A biodegradable tri-component graft for anterior cruciate ligament reconstruction. J Tissue Eng Regen Med 4: 2014.

41. Cooper J a, Lu HH, Ko FK, Freeman JW, Laurencin CT: Fiber-based tissue-engineered scaffold for ligament replacement: design considerations and in vitro evaluation. Biomaterials 26: 1523, 2005.

42. Lu HH, Cooper JA, Manuel S, Freeman JW, Attawia MA, Ko FK, Laurencin CT: Anterior cruciate ligament regeneration using braided biodegradable scaffolds: in vitro optimization studies. Biomaterials 26: 4805, 2005. 
43. Rothrauff BB, Lauro BB, Yang G, Debski RE, Musahl V, Tuan R: Braided and Stacked Electrospun Nanofibrous Scaffolds for Tendon and Ligament Tissue Engineering. Tissue Eng Part A: 2017.

44. Cho Y Il, Choi JS, Jeong SY, Yoo HS: Nerve growth factor (NGF)-conjugated electrospun nanostructures with topographical cues for neuronal differentiation of mesenchymal stem cells. Acta Biomater 6: 4725, 2010.

45. Liu HW, Chen CH, Tsai CL, Hsiue GH: Targeted delivery system for juxtacrine signaling growth factor based on rhBMP-2-mediated carrier-protein conjugation. Bone 39: 825, 2006.

46. Sahoo S, Toh SL, Goh JCH: A bFGF-releasing silk/PLGA-based biohybrid scaffold for ligament/tendon tissue engineering using mesenchymal progenitor cells. Biomaterials 31: 2990, 2010 .

47. Sahoo S, Ang LT, Goh JC, Toh SL: Growth factor delivery through electrospun nanofibers in scaffolds for tissue engineering applications. J Biomed Mater Res A 93: 1539, 2010.

48. Hankemeier S, Keus M, Zeichen J, Jagodzinski M, Barkhausen T, Bosch U, Krettek C, Van Griensven M: Modulation of proliferation and differentiation of human bone marrow stromal cells by fibroblast growth factor 2: potential implications for tissue engineering of tendons and ligaments. Tissue Eng 11: 41, 2005.

49. Kilian KA, Bugarija B, Lahn BT, Mrksich M: Geometric cues for directing the differentiation of mesenchymal stem cells. Proc Natl Acad Sci U S A 107: 4872, 2010.

50. Wells RG: The role of matrix stiffness in regulating cell behavior. Hepatology 47: 1394, 2008.

51. Subramony SD, Dargis BR, Castillo M, Azeloglu EU, Tracey MS, Su A, Lu HH: The guidance of stem cell differentiation by substrate alignment and mechanical stimulation. Biomaterials 34 : 1942, 2013.

52. Petrigliano FA, English CS, Barba D, Esmende S, Wu BM, McAllister DR: The effects of local bFGF release and uniaxial strain on cellular adaptation and gene expression in a 3D environment: implications for ligament tissue engineering. Tissue Eng 13: 2721, 2007.

53. Lin TW, Cardenas L, Soslowsky LJ: Biomechanics of tendon injury and repair. J Biomech 37: 865, 2004.

54. Cooper JA, Sahota JS, Gorum WJ, Carter J, Doty SB, Laurencin CT: Biomimetic tissueengineered anterior cruciate ligament replacement. Proc Natl Acad Sci U S A 104: 3049, 2007.

55. Bosworth LA, Rathbone SR, Bradley RS, Cartmell SH: Dynamic loading of electrospun yarns guides mesenchymal stem cells towards a tendon lineage. J Mech Behav Biomed Mater 39: 175, 2014.

56. Nerurkar NL, Sen S, Baker BM, Elliott DM, Mauck RL: Dynamic culture enhances stem cell infiltration and modulates extracellular matrix production on aligned electrospun nanofibrous scaffolds. Acta Biomater 7: 485, 2011. 
57. Teuschl A, Heimel P, Nu rnberger S, Griensven M van, Redl H, Nau T: A Novel Silk Fiber-Based Scaffold for Regeneration of the Anterior Cruciate Ligament: Histological Results From a Study in Sheep. Am J Sports Med, 2016.

58. Chen F, Hayami JWS, Amsden BG: Electrospun poly(1 -lactide-co -acryloyl carbonate) fiber scaffolds with a mechanically stable crimp structure for ligament tissue engineering.

Biomacromolecules 15: 1593, 2014.

59. Surrao DC, Fan JCY, Waldman SD, Amsden BG: A crimp-like microarchitecture improves tissue production in fibrous ligament scaffolds in response to mechanical stimuli. Acta Biomater 8: 3704, 2012.

60. Tozer S, Duprez D: Tendon and ligament: Development, repair and disease. Birth Defects Res Part C - Embryo Today Rev 75: 226, 2005.

61. Eriksen HA, Pajala A, Leppilahti J, Risteli J: Increased content of type III collagen at the rupture site of human Achilles tendon. J Orthop Res 20: 1352, 2002.

62. Amiel D, Frank CB, Harwood FL, Akeson WH, Kleiner JB: Collagen Alteration in Medial Collateral Ligament Healing in a Rabbit Model. Connect Tissue Res 16: 357, 1987.

63. Altman GH, Horan RL, Lu HH, Moreau J, Martin I, Richmond JC, Kaplan DL: Silk matrix for tissue engineered anterior cruciate ligaments. Biomaterials 23: 4131, 2002.

64. Fan H, Liu H, Toh SL, Goh JCH: Enhanced differentiation of mesenchymal stem cells co-cultured with ligament fibroblasts on gelatin/silk fibroin hybrid scaffold. Biomaterials 29: 1017, 2008.

65. Barry F, Boynton RE, Liu B, Murphy JM: Chondrogenic Differentiation of Mesenchymal Stem Cells from Bone Marrow: Differentiation-Dependent Gene Expression of Matrix Components. Exp Cell Res 268: 189, 2001.

66. Amiel D, Frank C, Harwood F, Fronek J, Akeson W: Tendons and ligaments: a morphological and biochemical comparison. J Orthop Res 1: 257, 1984.

67. Fan H, Liu H, Wong EJW, Toh SL, Goh JCH: In vivo study of anterior cruciate ligament regeneration using mesenchymal stem cells and silk scaffold. Biomaterials 29: 3324, 2008.

68. Spalazzi JP, Doty SB, Moffat KL, Levine WN, Lu HH: Development of controlled matrix heterogeneity on a triphasic scaffold for orthopedic interface tissue engineering. Tissue Eng 12: 3497, 2006.

69. Mosher CZ, Spalazzi JP, Lu HH: Stratified scaffold design for engineering composite tissues. Methods 84: 99, 2015. 


\section{Figure captions}

Figure 1: Aligned nanofibers (A), nanofiber bundle (B), scaffold composed of 20 nanofiber bundles (C), and small scaffold composed of 4 nanofiber bundles (D).

Figure 2: Chemical conjugation procedure (A), XPS survey spectra showing increasing nitrogen peak (B), immunofluorescence staining of conjugated CTGF (C), amount of CTGF conjugated (D), and CTGF release over a 14 day period (E).

Figure 3: 7 day in vitro nanofiber bundle CellTiter Blue assay results (A), representative SEM (B,D) and fluorescence $(C, E)$ images of cells on control $(B, C)$ and CTGF conjugated $(D, E)$ nanofiber bundles.

Figure 4: 21 day in vitro scaffold CellTiter Blue assay results (A), PicoGreen assay for DNA content (B), Day 21 hydroxyproline assay for collagen content (C), and Day 21 DMMB assay for sGAG content (D).

* Indicates significant difference between control and CTGF groups $(p<0.05)$. \# Indicates significant differences between Day 1 and Day $21(p<0.05)$.

Figure 5: Representative 21 day in vitro scaffold histology staining with picrosirius red for collagen $(A, B)$, safraninO/fast green for sGAG (C,D), and alizarin red for calcium (E,F) in control (A,C,E) and CTGF conjugated (B,D,F) scaffolds. Immunohistochemical staining for collagen type I $(\mathrm{G}, \mathrm{H})$ and collagen type III (I,J) for control (G,I) and CTGF conjugated (I,J) scaffolds. 
Figure 6: Representative 6 week in vivo histology staining with picrosirius red for collagen $(A, B)$ in control (A) and CTGF conjugated (B) scaffolds. Immunohistochemical staining for collagen type I (C,D) and collagen type III (E,F) for control (C,E) and CTGF conjugated (D,F) scaffolds. 
Figure 1

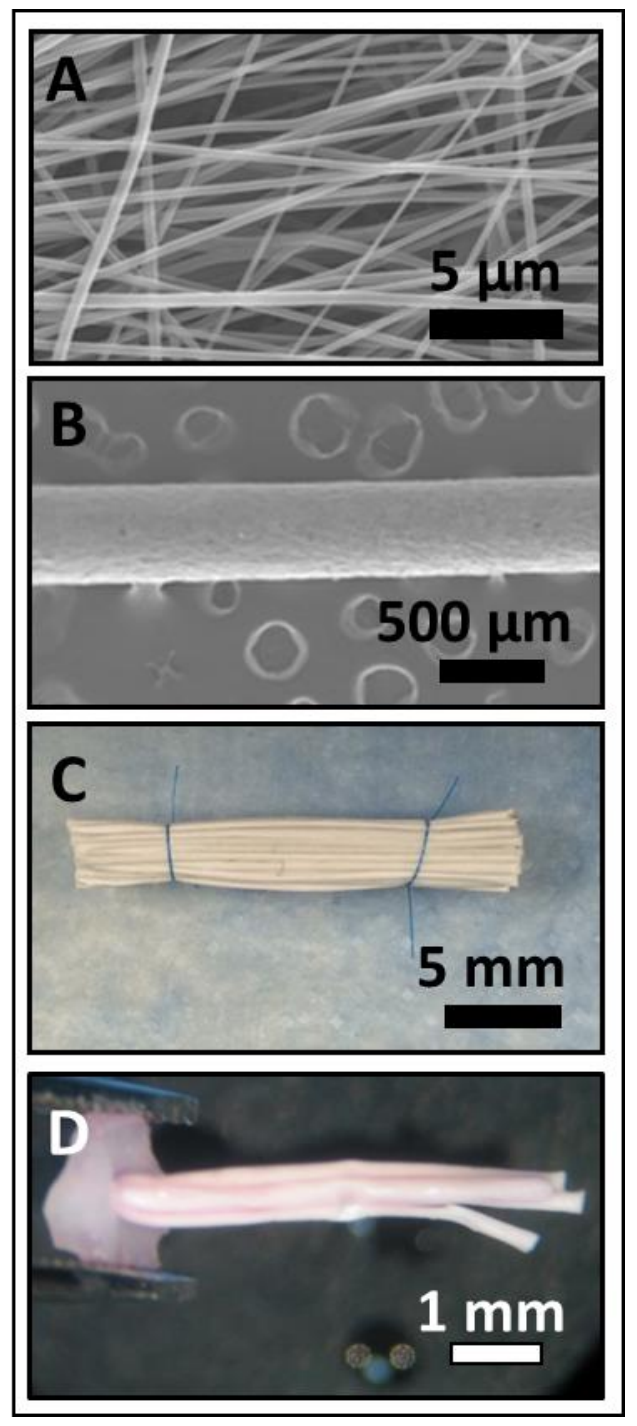


Figure 2

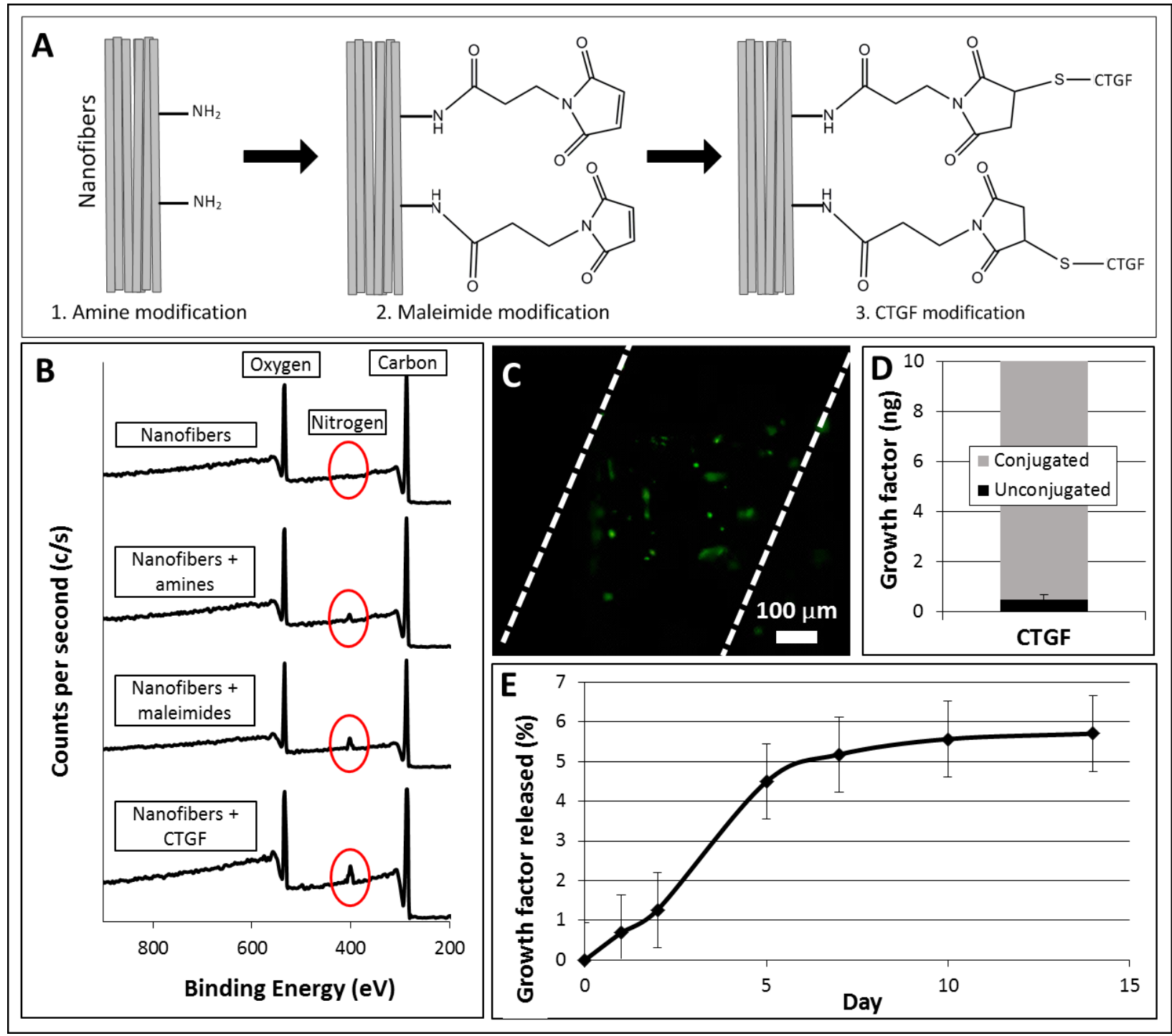


Figure 3

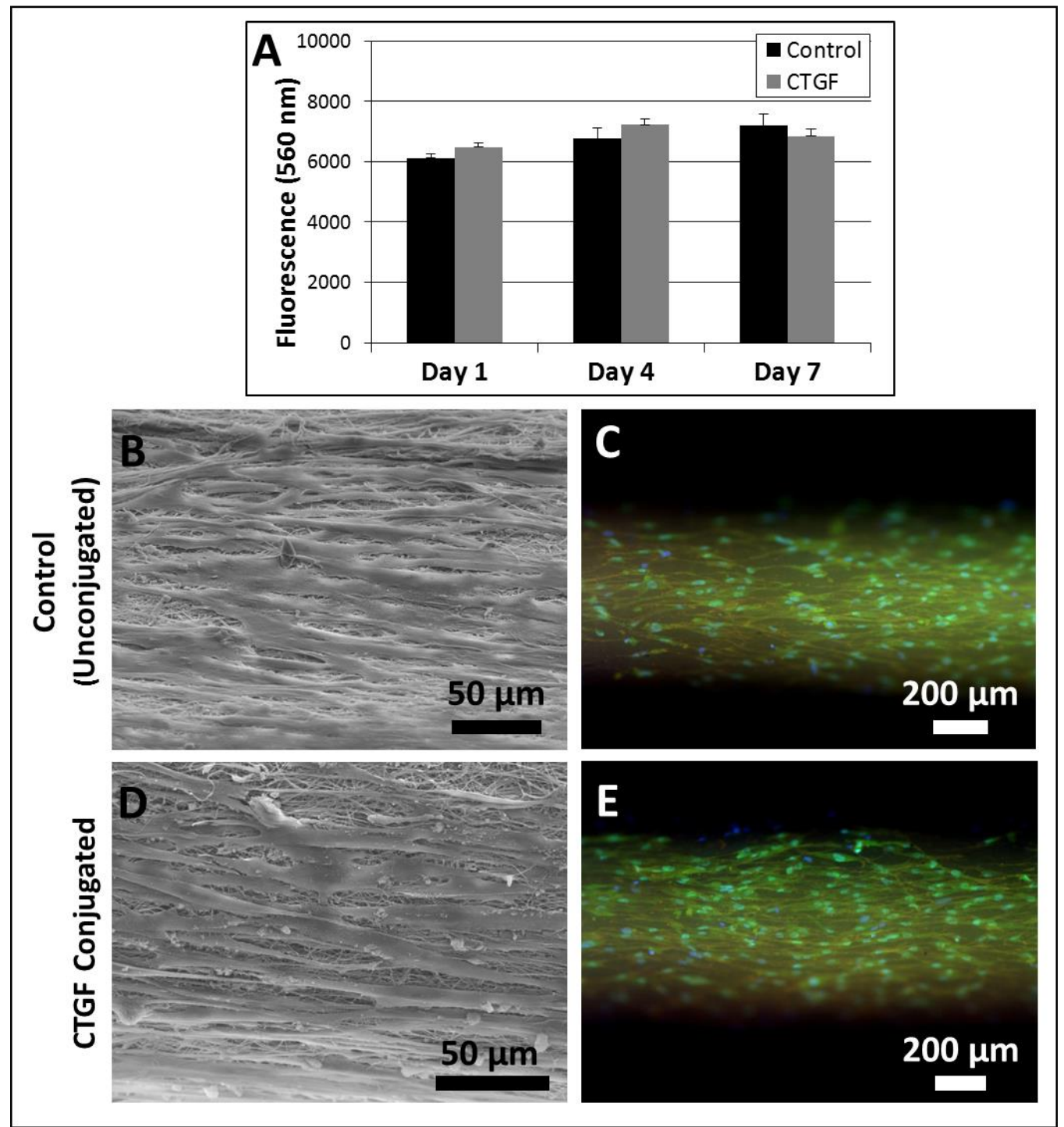


Figure 4

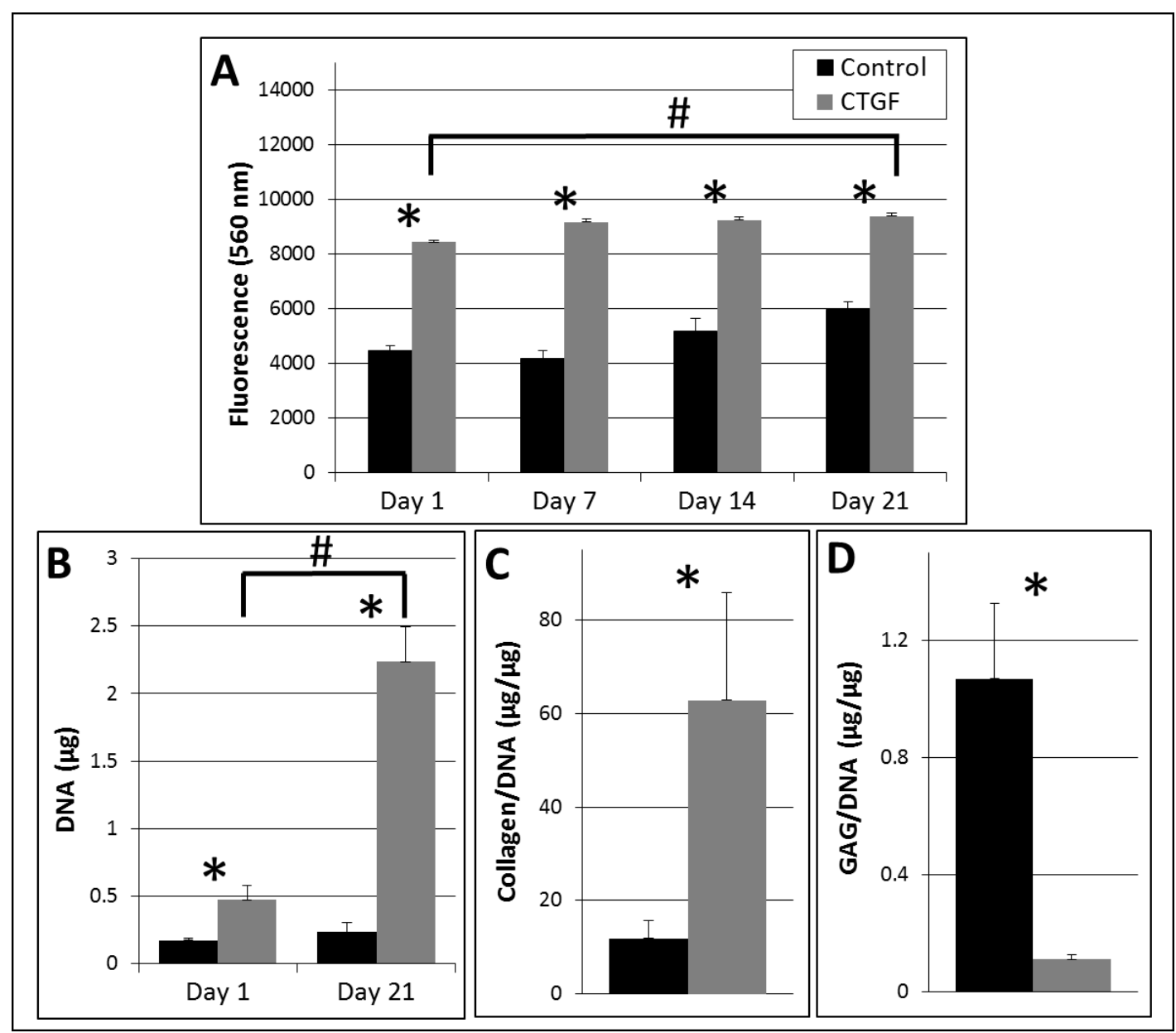


Figure 5

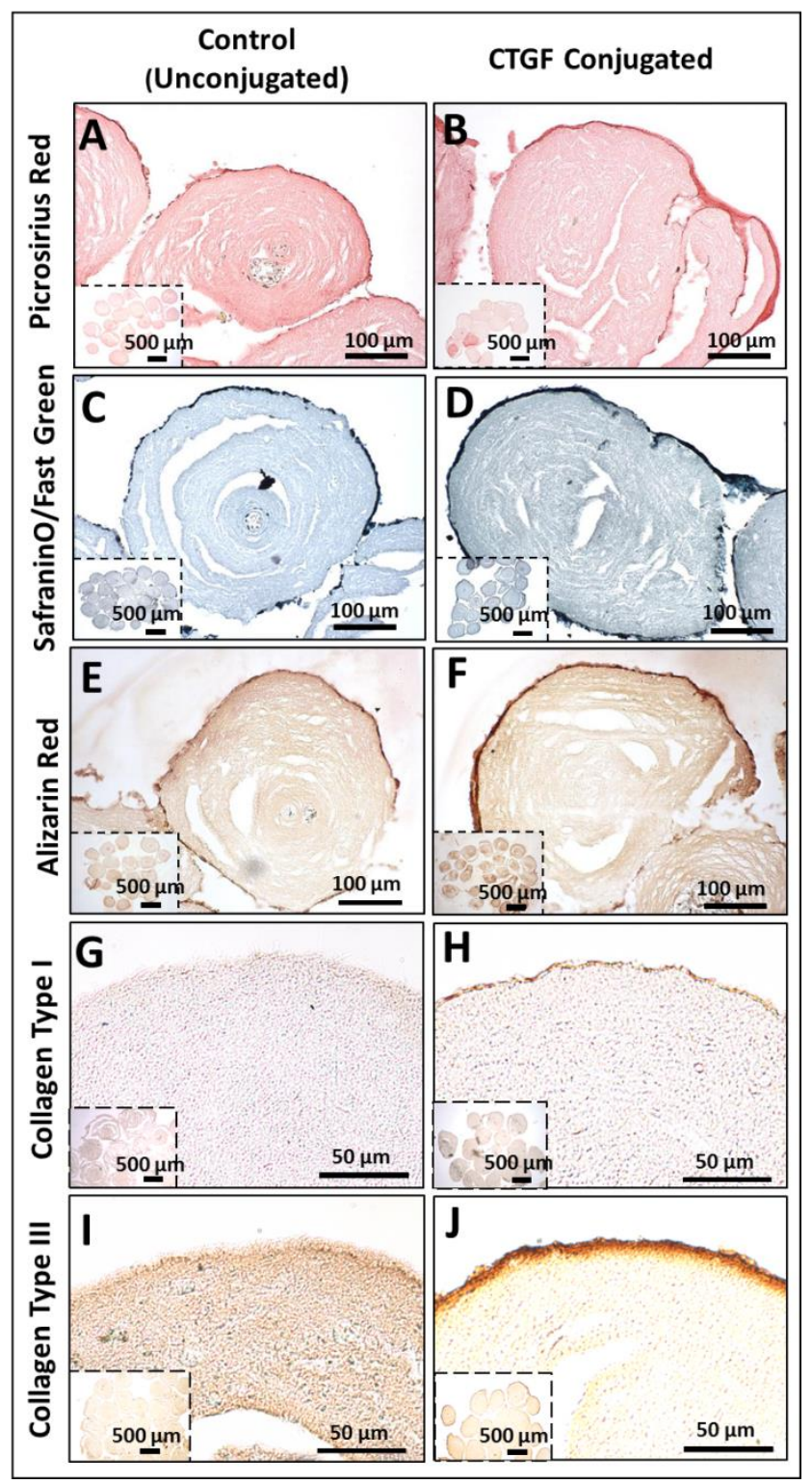


Figure 6

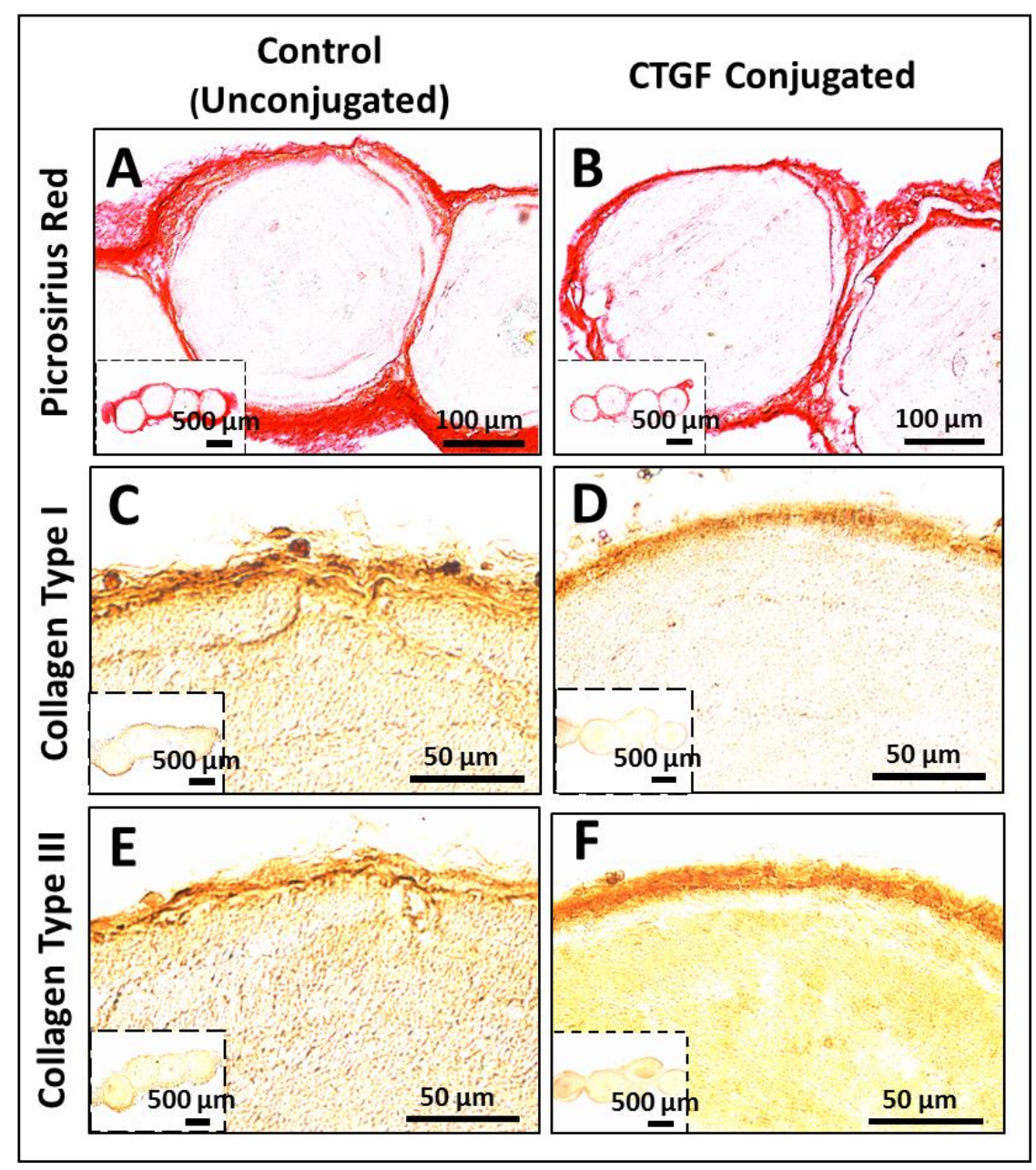

